\title{
The Hamburgers in the Fridge: An Interview with Professor Nikolas Rose about Interdisciplinary Collaboration, Neuroscience and Critical Friendship
}

\author{
By Kristofer Hansson and Karolina Lindh
}

During 2016 and 2017 the Cultural Studies Group of Neuroscience at the Department of Arts and Cultural Sciences at Lund University in Sweden organised a seminar series titled the 'Seminar on Neuroscience, Culture and Society'. Professor Nikolas Rose was one of the invited guest speakers; he is a researcher who strongly influences cultural reflections on neuroscience (Rose 2007, Abi-Rached \& Rose 2010, Rose \& Abi-Rached 2013). He visited us on the $22^{\text {nd }}$ of March 2017 and during his visit Kristofer Hansson and Karolina Lindh took the opportunity to interview Professor Rose to hear more about his thoughts and experiences of interdisciplinary collaboration between neuroscience researchers and researchers in the social sciences and humanities.

Question: Today you find neuroscientific knowledge almost everywhere, not only in the laboratory. You have written extensively about this, and in your article "The Human Sciences in the Biological Age" (2013), you write that social scientists and medical scholars should engage in a critical friendship. We would like to know a little more about this; how do you envision such a friendship?

Nikolas Rose: There have always been psychological explanations and arguments about the brain, circulating outside the scientific literature. The real move of neuroscience out of the labs happens in the 1990s and 2000s, when some neuroscientists start to argue that they have expertise that can be relevant in a whole range of different disciplines. In a sense, these are all those disciplines that psychologists have already colonized, if you want to put it like that. Some neuroscientists argue that they can give more objective explanations or more objective underpinnings of explanations in those domains-the obvious one is clinical psychiatry-but you see this in education, legal system and in a range of other places.

Hansson, Kristofer \& Karolina Lindh: "The Hamburgers in the Fridge: An Interview with Professor Nikolas Rose about Interdisciplinary Collaboration, Neuroscience and Critical Friendship", Culture Unbound, Volume 10, issue 1, 2018: 115-122. Published by Linköping University Electronic Press: http://www.cultureunbound.ep.liu.se 
Sometimes it is entrepreneurial individuals who want to make the claim to have impact, certainly in the UK and in the United States. But these days the argument that your research has impact in the real world is central to doing well in assessments for promotion at Universities, and central to getting research funding. You have got to say that your research is having or will have an impact. The good side of that may be that neuroscientists are directing their attention towards important practical issues, but the bad side of it may be that neuroscientists are tempted to make exaggerated claims about the implications of their work for domains that they do not really understand. I think the legal system is one of those domains. Arguments about volition and free will have always played a key part in the criminal justice system, and some neuroscientists began to argue that because of the unconscious shaping of apparently willful volitional actions, this should really radically transform the way in which the criminal justice system operated. This wasn't only a very unwarranted extrapolation from the laboratory experiments to real-life situations, but it showed considerable ignorance about how legal systems worked.

So, the critical bit of the critical friendship is to try and inject a little more realism into these extrapolations, especially from clinical experiments or laboratory experiments with animals. The friendship side of it is to say that there is nothing for social scientists to be afraid of in the emergence of neurobiological accounts of human conduct. It is not as if, the limits of how it is possible to understand human beings come from psychology, which is folk psychology of sociologists - they mostly just think of human beings as psychological creatures with wishes and intentions and biographies and so forth. You will know, if you have done the history of psychology, that all those beliefs about what humans are like are quite recent, dating back to the 19th century. So, there is no reason why social scientists should say that "well, that's the right way to understand human beings and any other way is a great threat to that". The friendship is to try and encourage those kinds of relationships, the critical side is to do this without the over-claiming the neuroscientists often do.

But the more epistemological part is based on an assessment of how neuroscientists do their work. Because, since the emergence of neuroscience as a kind of label, in the 1960s, the argument was always that neuroscience should start by trying to understand the very basic components of the most simple systems, the most simple organisms at the most basic level. To take Eric Kandel's work on memory, done with the Californian sea slug that has about twenty thousand rather large neurons, is one example of this kind of neuroscientific research. You can make this Californian sea slug learn something-when its gill withdrawal reflex is repeatedly provoked, it will 'habituate-and then you think you are understanding something about the basic elements of memory. Your challenge is 
then to scale that up from the sea slug to the rat, from the rat to the macaque, from the macaque to the higher primate and from the higher primate to the human; and from the single neuron to the billions of neurons and synapses in the human brain. It was almost as if that scaling up was a technical task, whereas I argue that it is the wrong way to conceive of the issue. This is a more difficult kind of critical friendship, because it is criticizing some very basic assumptions of experimental neuroscience. It is arguing that whatever you understand at this reductionist level, you need to recognize that this is only a first step, and that you need to put it back into the organism as a whole; and you have to understand how it works in the organism as a whole. This is not a novel thought! Claude Bernard, in his book on experimental medicine, agues exactly that; the researchers should never ever forget that if they are isolating things in the laboratory, what they really are trying to understand are complex organisms in their world. That is the more difficult part of the critical relationship because it requires not just a rethinking of experimental practice, but a rethinking of the object-the object that the experiment is directed towards.

Question: It is really interesting to see the two terms critical and friendship together. We have this long history in the social sciences and the humanities with critique; for example Ivan Illich and Michel Foucault. Then we have the term friendship that we see more as a metaphor. We think that you point out that we in the social sciences and the humanities should also engage in this kind of friendship.

Nikolas Rose: Yes, I suppose it depends how attracted one is to the posture of critique. I think what Foucault did was more description than critique. I would like to think that that kind of work, genealogical work, is not itself critique, it is designed to make criticism and critique possible. Because you uncover the way in which the machinery, the apparatus, the complex sets of connections amongst things work; what they bring into existence and how they have emerged just directly; how they function, what the consequences are. Once you have begun to describe that set of relations, how they come about and what they produce, you are in a position to make critical evaluations of them and these are in many ways political and ethical evaluations about the kinds of people you would like us to be, the kinds of things you think are good for humans and so on. But they don't spring fully formed out of the description. It is a bit Weberian, you go into studying an area because you are passionate about a set of problems, but you leave your passion at the door in order to understand what is going on; then you bring the passion back in the end. Just continually saying what is wrong with things and how naive people are, and how scientists do not really understand their own assumptions-I do not find that a particularly appealing posture. I would also 
argue that most work in the life sciences is probably more sophisticated, more subtle, more difficult to achieve than the work of most social scientists. It is quite difficult to makes things true in the life sciences. In social sciences it's not so hard to make things true if you have a lot of convincing rhetoric and you can mobilize enough supporters in the circles around you. This is why I think it is necessary to go beyond critique. The friendship bit of it, is to say that the questions that the researchers in the life sciences are trying to understand are often important questions. One might want to work with them to understand those questions. To try and work on the assumption that they are well-meaning individuals just the same as you are, rather than to start from a hermeneutics of suspicion about what they are doing, that they are only in for the money, for the influence, for the research papers and so on. They probably are in it for all those things as well, but no more than the social scientists.

Question: How do you work with medical researchers in a more practical way? What does your research practice with medical researchers look like?

Nikolas Rose: There are many different ways of doing it. I have students, especially research students who work as anthropologists with life scientists of various sorts, for instance with people who work in synthetic biology. They have labs and my research students go and embed themselves in the labs, they sit in and they take on some roles, they do some of the work and they get trained up in various ways. They sit in the lab meetings but they are not just passive observers, they to ask people questions like: why do you think that is important? Why are you doing that? What is the role of that experiment? How does that experiment link to this experiment? Why is this specific part so important to understand? In particular in some of these emerging disciplines, there are still many un-answered questions and different points of view and actually some of the researchers find it rather useful to be asked to think and speak explicitly about these issues.

In the current research that I am doing, I try and work with life scientists right from the beginning of developing of the research. In the work we are doing on mental ill-health in cities, for a whole range of reasons, stress was one of the issues that kept coming up, so we started reading some work on stress. We identified the person who was working in my institution, who was the guy most enthusiastic about stress research, and we went and talked to him about the research that we were doing. Partly to learn the protocol that he was using to measure cortisol levels as an index of stress, partly to see what he felt about the experiment that we were doing. He became interested in this experiment and became a collaborator in the research. Although we have not got to the stage now of writing the papers, we will probably co-author some of the papers. The same with how we are developing 
this app to assess mental states in city space and time. The app was developed by someone in an early intervention psychosis unit to monitor the mental state of those who had a first psychotic episode. We saw the app, we thought it would be very good to monitor mental states in the migrant populations that we were studying, and we went to talk to him. We started to work with designers; they are interested in space and how space might affect people's experience of being in cities. Our psychiatrist colleague is usually a lab researcher; he is finding it kind of fascinating to think about these broader issues. But people thinking of these broader issues are also fascinated to think about how this stuff gets into the brain.

So, if you can get people excited about something, you work together. It does not mean that you will not argue about it; especially about methodology! The first question most scientists ask is: "This is the experimental group, so where is your control group? How much data are you going to have? How many variables have you got? If you have got those number of variables, you need to have at least this number of subjects in your experimental group, and at least these number in your control group, in order to power up the statistics". Those of us who are into sort of street level of ethnography think that "oh no there is no way we are going to get something like that, 500 subjects and 500 controls". We then have interesting discussions about whether it is possible to get any data that is robust data from these kind of quasi experimental technologies, quasi experimental research designs.

It is also relevant, that how you work across the disciplines differs according to, to be crude about it, agent status. For a young social scientist, it is quite prestigious to be able to work with a life scientist and publish in a collaborative way. For a post-doc life scientist, it's not at all prestigious to work with a social scientist and publish in "Body and Society" or something like that. They are not going to get their tenure because they published in "Body and Society"; so there are real differences. When you are senior in your career and you have nothing to lose, then it is easier to collaborate. You know, you don't need to build up your $\mathrm{CV}$, you are not really worried about getting another big grant or something like that. You are free to experiment in a way that you are probably not if you are at an earlier stage in your career.

Question: But if there is no friendship?

Nikolas Rose: Then there is no collaboration. If people do not trust one another and they do not find it interesting to talk to one another and they do not feel that they can talk frankly about the problems without it immediately becoming critique. 
Because there are many problems, as we, as anybody who has done research knows. From the very beginning you run into all sorts of problems, you make all sorts of mistakes. You try and learn from your mistakes and correct things, but it does not look at all like it looks when you write it up for the article, you know it is a much messier process. Unless you can begin to talk about that frankly, without thinking that whoever you are talking to is going back and talking to their friends saying, "Oh do you know professor so and so, he really does not understand $\mathrm{x}$, $y$ and z". Unless you can have a relationship of trust, then it is not going to work. Certainly, for the medics and the life scientists, you are pulling people out of their comfort zone, you are pulling them out of what they know. And you are pulling them away from the things that they know are going to be the conventional ways of advancing their research trajectory and their research career. There has got to be something interesting and enjoyable for them to be doing there. Whereas most social scientists think it is a good thing to be working with a life scientist, it is fun and enjoyable and we are studying them. They usually do not like being studied! They do not like being our rats! In one of the very first collaborations I was in with some geneticists, I was trying to make them understand why they might want to work with us, and the person who was in charge of the genetic side, she said, "Well, what he is trying to say is that we are his rats!" They thought that was funny but they did not like being the rats.

Question: During your lecture yesterday, you were talking about labs and experiments and we were seeing that as metaphors, how you were talking of trying to do new things without knowing whether it will turn out right or not. We really liked the metaphors of seeing ourselves as doing some form of experiments or being in lab or so. Could you elaborate a little bit on that?

Nikolas Rose: I think there are experiments in a number of different ways, but I do not think they are entirely metaphors. It depends on what you mean by an experiment. So, part of the experiment is this: is it possible for a group of social scientists and a group of life scientists to come to a common understanding of what a problem is, a common agreement as to a set of research protocols and a common agreement as to how the data should be collected, interpreted and analyzed. To some extent, this gets to be formalized: if you are going to do an experiment or doing any work which involves human subjects, you have got to put it through an ethics committee. And if you put it through an ethics committee, you have got to make a lot of the things about your protocols explicit, so the group actually has to sit down together and say "right, are we all prepared to sign up, we are doing this, we are doing this, we are doing this and we are doing this.... Is it possible for people to actually agree? In our experiment we have seen that it is possible 
for people to agree, as far as getting the protocols and the empirical research are concerned. But we do not yet know whether it is possible to get people to agree when you get the different findings together, as to which findings are important and which are not important, which bits you can discard. This is partly because we have got multiple methods going on. So that bit is experimental. And then, I suppose, the whole approach is experimental. Is it possible to take this idea of a collaborative biosocial or sociobiological approach to a question, to a problem to operationalize it, to carry out a piece of research, to draw conclusions which have some practical consequences? Is it actually possible to do that? It is possible to do a thought-experiment that you can do it. It is possible to write a manifesto that says; "It should be done!" But whether it is possible to take a particular question and make it researchable and do the research; that I do not know.

Now, I do know from years and years ago, when I was a baby biologist, that, especially if you are a baby biologist and you are trying to do experiments in the lab, they fail all the time. You have not got the reagents right, it is the wrong temperature, you have put it in the wrong place, somebody turned off the fridge, you forgot it was a long weekend and you came back on the Tuesday rather than on the Monday and everything had gone to hell and somebody had left a hamburger in the fridge because they were going eat it the next day and it polluted everything. All you know is that your experiments failed. You try and find the different reasons, was it the hamburger in the fridge, was it the wrong reagent? Then you try it again, and gradually you begin to learn what makes a difference and what does not make a difference. And if you are fortunate you make the thing work, but you do learn from the failures. You do learn, "Ah, this reagent only works if you have the temperature between 18 and 22 degrees. If it is 15 degrees it is not going to work and if it is 24 degrees it is not going to work". So, you learn something, that the concentration of the bugs has to be like this, etc. All of this which is usually quite tacit knowledge, which you acquire in becoming a skilled experimenter: you know the craft work of doing it. You learn from the failures. To be a sociologist you also go through a long period of training. My sociology students, they start going out in focus groups and things like that in the first year. They design questionnaires and the questionnaires fail because they have leading questions and things like that; over four years they learn through their failures how to do it. It would not be surprising, that in even more complex situations when you are using the techniques from different sorts of disciplines, a lot of the time it is going to fail. "Oh, we did not realize we could not make the correlation between what you were getting in this rating scale and what you were getting in the app because they were using different definitions of stress" or whatever it happens to be. But you hope to learn; it is not trivial to say that you learn from the failures. 
Question: Do you think that if we use the hamburger and the fridge, as an example, do you think that we can come together as social scientists and natural scientists to focus on our mistakes?

Nikolas Rose: I hope we are not the hamburgers in the fridge! I think as long as social scientists realize that when they identify the craftwork that is required to make an experiment in biomedicine work, that that is not a critique of the experiment, the craftwork is how you make the experiment work. As long as we are prepared to engage and not be ironic all the time about it. As long as we are prepared to do it genuinely, to actually think together. It also depends on whether there are questions or problems that you think can only be adequately understood through this collaboration. I do not think it is something that you should do just for its own sake, because it is good to collaborate, "oh let us collaborate". I do not think that is a good reason for doing it. But I think that if there are specific questions of importance that can only be understood by bringing these things together, then it is worth doing. I personally think that questions of mental ill health can only be understood that way. Then it is worth trying to do it and see what happens.

Question: Thank you for your time and thank you for answering our questions!

Nikolas Rose: Thank you!

\section{References}

Abi-Rached, Joelle M. \& Rose, Nikolas (2010): The birth of the neuromolecular gaze, History of the Human Sciences, 23: 1, 11-36.

Rose, Nikolas (2007): The Politics of Life Itself: Biomedicine, Power, and Subjectivity in the Twenty-first Century, Princeton: Princeton University Press.

Rose, Nikolas (2013): 'The Human Sciences in a Biological Age', Theory, Culture \& Society, 30:1.

Rose, Nikolas \& Abi-Rached, Joelle M. (2013): Neuro: The New Brain Sciences and the Management of the Mind, Princeton: Princeton University Press. 